framework.

The analysis of performance over time offered little of interest other than the information that differences appeared early and remained throughout the experimental session.
REFERENCE

COTTRELL, N. B. Performance in the presence of other human beings. Mere presence, audience, and affiliation effects. In $\mathrm{E}$. C. Simmel, R. A. Hoppe, and G. A. Milton (Lis.) Social facilitation and imitative behavior. Boston: Allyn \& Bacon, 1968. Pp. 91-110.

\section{Words functioning simultaneously as negative operant reinforcers and unconditioned stimuli}

\author{
ANDREW T. ABELL* \\ Southeastern Louisiana College, Hammond, La. 70401
}

To continue an analysis of earlier findings, data were secured from 112 undergraduates in a study employing an identical Ss design. A conditioning device made of paper (reinforcer form) allowed exposures of unpleasant words and of neutral ones to be dependent upon motor responses to two CVCs. As predicted, Ss tended to expose neutral words rather than unpleasant ones, and they subsequently rated the $\mathrm{CVC}$ associated with unpleasant words as less pleasant than the other trigram.

This study continues an analysis (Abell, 1970) of earlier results (Abell, 1969a, b, c). Abell (1970) recently demonstrated the contemporaneous operation of pleasant words as UCSs and positive operant reinforcers, thereby revealing one source of earlier findings (Abell, 1969a, b, c). The present study was designed to determine whether or not unpleasant words can function simultaneously as UCSs and suppressors of operant behavior. Confirmation would indicate that words functioning simultaneously as negative operant reinforcers (via punishment) and as UCSs also contributed to the earlier results (Abell, 1969a, b, c).

The hypotheses were the following: (1) The tendency to respond to CVCs followed by unpleasant words is less than that to CVCs followed by neutral words; (2) CVCs preceding unpleasant words are rated as less pleasant than those preceding neutral words.

\section{SUBJECTS}

Students in seven introductory psychology classes (56 males and 56 females) served as Ss during regular class periods. They were given no advance information about the experiment.

$$
\text { MATERIALS }
$$

Two CVCs, XIB and ZUJ, which have the same scaled meaningfulness (Noble, 1961), and eight evaluative words, were

*Beginning September 1970, at Westminster College, New Wilmington, Pa. 16142. presented in an identical Ss design by means of reinforcer forms like those used earlier (Abell, 1970). The evaluative words, four neutral and four unpleasant, were chosen from previous ratings on a 7-point scale (Abell, 1970, Table A). The neutral words were the same four used before (Abell, 1970). The unpleasant words, together with their medians $(1.000$ represents the extreme of unpleasantness), are the following: agony, 1.245; vomit, 1.245; terror, 1.391; and failure, 1.411. As in earlier studies (Abell, 1969a, b, c, 1970) a rating sheet was included with each reinforcer form.

As before (e.g., Abell, 1970), the sets of materiais incorporated various controls. In half the forms, XIB concealed an unpleasant word and ZUJ, a neutral one (Tab Placement $\mathrm{U}$ ); in the others, the reverse was true (Tab Placement N). These different tab placements, together with two different arrangements of words on undersheets (A and B) and two slightly different rating sheets ( 1 and 2), produced eight different booklets: U-A-1, U-A-2, U-B-1, U-B-2, N-A-1, N-A-2, N-B-1, and N-B-2.

\section{PROCEDURE}

As the design required a multiple of eight Ss per class, from four to seven students were eliminated in a random way from each class. Then, the eight different kinds of booklets, which had been stacked earlier in a sequence randomized within each block of eight, were distributed by rows to the remaining students. Thus, a total of $14 \mathrm{Ss}$ was assigned to each of the eight control conditions.

As in earlier studies (Abell, 1969a, 1970), Ss were instructed to use a recorded sequence of words ("open," "choose," and "close") to pace responses to the forms; they were allowed about a minute's rest between pages; and they subsequently rated the CVCs for pleasantness on a 7-point scale.

RESULTS AND DISCUSSION

Neutral words were exposed more often than unpleasant ones by $71 \mathrm{Ss}$ and less frequently, by 34 ; the two types were exposed equally often by 7 . The sign test for large samples (Siegel, 1956) produced a $\mathrm{z}$ of 3.32 (one-tailed $\mathrm{p}<.0005$ ). The CVC associated with unpleasant words was judged, subsequently, as less pleasant than the other one by $63 \mathrm{Ss}$ and as more pleasant, by 29 ; the two were evaluated the same by 20 . The sign test yielded a $z$ of 3.44 (one-tailed $\mathrm{p}<.0003$ ).

The confirmation of both hypotheses demonstrates that words can function simultaneously as - negative operant reinforcers and UCSs. This demonstration, together with the one that showed that words can function concurrently as positive operant reinforcers and UCSs (Abell, 1970), helps explain earlier results (Abell, 1969a, b, c). Apparently, both positive reinforcement and punishment operated to produce the difference in strength of operant response to trigrams that was found in the initial (Abell, $1969 \mathrm{~b}$ ), as well as in each confirming (Abell, 1969a, c), gross-comparison study. Similarly, the difference in evaluative meaning of trigrams that was found in those early studies (Abell, 1969a, b, c) seems to reflect the operation of two processes: (1) the conditioning of pleasantness to one of the trigrams; (2) the conditioning of unpleasantness to the other.

\section{REFERENCES}

ABELL, A. T. A test of reinforcer forms with flaps. Perceptual \& Motor Skills, 1969a, 29, 984-986.

ABELL, A. T. Words functioning simultaneously as operant and respondent reinforcers: Preliminary study. Psychological Reports, 1969 b, 24, 123-133.

ABELL, A. T. Words functioning simultaneously as operant and respondent reinforcers: Replication. Psychological Reports, 1969c, 25, 975.

ABELL, A. T. Words functioning simultaneously as positive operant reinforcers and unconditioned stimuli. Psychonomic Science, $1970,19,325-326$.

NOBLE, C. E. Measurements of association value (a), rated associations (a'), and scaled meaningfulness $\left(\mathrm{m}^{\prime}\right)$ for the 2100 CVC umbinations of the English alphabet. Psychological Reports, 1961, 8, 487-521.

SIEGEL, S. Nonparametric statistics for the behavioral sciences. New York: McGraw-Hill, 1956. 